\section{Postanalytische Phase}

W. G. Guder

München, Deutschland

Synonym(e) Postmetrologische Phase

Englischer Begriff postanalytical phase; postmetrological phase

Definition Die postanalytische Phase laboratoriumsdiagnostischer Prozesse umfasst alle Prozesse und ihre technischen und geistigen Inhalte, die zwischen der Erstellung des Messwerts und der medizinischen Entscheidung auf der Basis des Befunds ablaufen.

Beschreibung Nach Ablauf der analytischen Phase liegt ein Messwert vor, der auf technischer, biologischer und nosologischer Ebene zum interpretierten $>$ Befund wird und als solcher Grundlage für diagnostische, prognostische oder therapeutische ärztliche Entscheidungen ist. Dabei sind folgende Prozesse zu berücksichtigen:

- Technische Ebene: formale Kontrolle (Identität, Material, System), analytische Beurteilung inkl. Qualitätskontrolle
( $\triangleright$ Qualitätskontrolle, statistische), statistische Beurteilung möglicher analytischer $>$ Störgrößen

- Biologische Ebene: Beurteilung von Einflussgrößen, Plausibilitätskontrolle, Transversalbeurteilung (z. B. Vergleich mit Normalbereich), Longitudinalbeurteilung (Vergleich mit Vorwerten)

- Nosologische Ebene: pathophysiologische Beurteilung, Bewertung von $>$ Einflussgrößen beim Patienten, Zuordnung zu definierten Krankheiten und Entscheidungsgrenzen, differenzialdiagnostische Beurteilung

Diese Prozesse können von kompetenten Laboratorien bis zur biologischen Ebene durchgeführt werden und führen so vom Messwert zum Befund. Die nosologische Ebene ist normalerweise nur mit Kenntnis weiterer Informationen zum Patienten, Ergebnissen anderer Untersuchungen und der Krankengeschichte durchführbar und ergibt den interpretierten Befund, der als Grundlage medizinischer Entscheidungen dient.

\section{Literatur}

Wisser H, Bertsch T (2009) Aussage und Nutzen von Laborergebnissen. In: Guder WG, Nolte J (Hrsg) Das Laborbuch für Klinik und Praxis, 2. Aufl. Elsevier/Urban und Fischer, München, S 21-38 